\title{
A comfort comparison of travoprost BAK-free $0.004 \%$ versus latanoprost $0.005 \%$ in patients with primary open-angle glaucoma or ocular hypertension
}

\author{
David A Godfrey' \\ Lee S Peplinski \\ Jeanette A Stewart ${ }^{3}$ \\ William C Stewart ${ }^{3}$ \\ 'Glaucoma Associates of Texas, Dallas, \\ TX, USA $;{ }^{2}$ Kentuckiana Institute for \\ Eye Research, Louisville, KY, USA; \\ ${ }^{3}$ PRN Pharmaceutical Research \\ Network, LLC, Dallas, TX, USA
}

Purpose: To determine the short-term comfort after a single dose of travoprost BAK-free compared to latanoprost in primary open-angle glaucoma or ocular hypertensive patients.

Design: Prospective, double-masked, randomized comparison of two separate active agents dosed once in opposite eyes.

Methods: At Visit 1, qualified patients began a glaucoma medicine-free period for three days. At Visit 2, patients were randomly assigned to travoprost BAK-free or latanoprost in opposite eyes. Following dosing in each eye, patients completed a visual analog scale (VAS score, 0-100 mm) at specified time intervals and a comfort survey.

Results: In 54 completed subjects, no difference existed five seconds after dosing, in comfort on the VAS between latanoprost $(7.1 \pm 16.2 \mathrm{~mm})$ and travoprost BAK-free $(7.8 \pm 16.1 \mathrm{~mm}$, $\mathrm{P}=0.53$ ). Also no differences existed between treatments following dosing for discomfort at individual timepoints past five seconds, peak discomfort or the time required to return to baseline comfort $(\mathrm{P}>0.05)$. In addition, the comfort survey demonstrated no difference between products for burning, stinging, foreign body sensation, overall comfort and general acceptance between the products, both for absolute levels and changes from baseline ( $\mathrm{P}>0.05)$.

Conclusion: Following a single instillation, both latanoprost and travoprost BAK-free exhibit similar comfort scores.

Keywords: comfort, travoprost BAK-free, latanoprost, glaucoma, ocular hypertension

\section{Introduction}

Glaucoma patients are typically treated medically to lower the intraocular pressure to prevent glaucomatous progression. ${ }^{1}$ Unfortunately, patients may have irritating concomitant anterior segment diseases such as lid lag, blepharitis or dry eye, in which the use of a glaucoma preparation, which usually includes both a preservative and an active ingredient, might further exacerbate their symptoms., ${ }^{2,3}$

Several years ago, Allergan made available brimonidine purite (Alphagan PTM, Allergan, Inc., Irvine, CA), a chronically dosed benzalkonium chloride (BAK)-free alpha-agonist, which eliminated the BAK preservative. Although the medicine appears equal in efficacy to BAK-preserved brimonidine, little evidence exists that it improves comfort. $^{4}$

More recently, Alcon released travoprost BAK-free (Travatan Z ${ }^{\mathrm{TM}}$, Alcon Laboratories, Inc., Fort Worth, TX) which is a chronically dosed BAK-free prostaglandin. This formulation is preserved with Sofzia ${ }^{\mathrm{TM}}$, which provides an antimicrobial effect through a proprietary formulation of several buffering agents. ${ }^{5}$ Regulatory trials have indicated equivalent efficacy between travoprost and the preservative-free formulation. ${ }^{5}$ 
Unfortunately, little information yet is available evaluating comfort with travoprost BAK-free. Data might be especially important compared to latanoprost (Xalatan ${ }^{\circledR}$, Pfizer, New York, NY) which is the leading selling glaucoma agent, but also has manifested few comfort problems. ${ }^{6,7}$ The primary objective of this study was to determine the short-term comfort after a single dose of travoprost BAK-free compared to latanoprost in patients with primary open-angle glaucoma or ocular hypertension.

\section{Materials and methods}

\section{Patients}

The study design was a prospective, double-masked, randomized comparison of an active agent in one eye and the active control in the opposite eye (intra-individual control).

We included patients with a clinical diagnosis of ocular hypertension or primary open-angle, pigment dispersion or exfoliation glaucoma with an intraocular pressure considered to be safe, in both eyes, for a 3-5 day washout from current medical therapy. Patients must have demonstrated best corrected Snellen visual acuity of 20/200 or better in each eye and their intraocular pressure must have been $\leq 22 \mathrm{mmHg}$ in both eyes while treated with any topical ocular prostaglandin or $\leq 30 \mathrm{mmHg}$ on no medications.

We excluded patients who had a presence of other primary or secondary glaucoma not listed in the inclusion criterion; any abnormality preventing reliable applanation tonometry; any known opacity or patient uncooperativeness that restricted adequate examination of the ocular fundus or anterior chamber in either study eye; or a concurrent infectious/noninfectious conjunctivitis, keratitis or uveitis in either eye. Blepharitis, nonclinically significant, or prostaglandininduced conjunctival injection was allowed.

We also excluded patients who had undergone intraocular conventional surgery or laser surgery less than three months prior to Visit 1; were at risk of visual field or visual acuity worsening as a consequence of participation in the trial; had progressive retinal or optic nerve disease from any cause; demonstrated any clinically significant, serious, or severe medical or psychiatric condition; had any condition which would interfere with participation in the study or which would present a special risk to the patient; demonstrated known allergy, sensitivity or poor tolerance to any components of the preparations used in this trial; had a history of, or were at risk for uveitis or cystoid macular edema; had a history of ocular herpes simplex; or required use of ocular or oral corticosteroids, contact lenses or topical ocular cyclosporine currently or within the past 30 days.

\section{Procedures}

At Visit 1, patients signed an Institutional Review Board informed consent form. Patients then underwent an examination which included: Goldmann applanation tonometry, Snellen visual acuity, and slit lamp biomicroscopy, and completed the Ocular Surface Disease Index (OSDI) survey. ${ }^{7}$ Patients whom the investigator believed could safely stop their current glaucoma medications for 3-5 days, and met the inclusion/exclusion criteria, were enrolled into the study. Patients were instructed to stop their glaucoma medication(s) at the end of Visit 1.

Visit 2 (Day 1) occurred 3-5 days or longer after Visit 1. The washout period was not designed to obtain an accurate baseline for the intraocular pressure but to allow for normal turnover of epithelial cells on the anterior surface of the eye to assess comfort. ${ }^{7}$ Patients again underwent visual acuity testing and slit lamp biomicroscopy. Patients also completed a baseline visual analog scale, which evaluated global comfort, and a comfort survey which assessed specific anterior segment side effects. Afterwards, patients were randomly assigned to which eye would receive medicine first and which eye was dosed with travoprost BAK-free (Travatan $\mathrm{Z}^{\mathrm{TM}}$, Alcon Laboratories) or latanoprost (Xalatan ${ }^{\mathrm{TM}}$, Pfizer).

Patients were randomized to the masked study medicine and were dosed by an unmasked dosing coordinator. Immediately following dosing of one drop of the study medicine in the first eye, patients completed the visual analog scale (VAS) at every five seconds up to one minute and then at $2,5,10,15,20$, and 30 minutes, or until comfort returned to baseline ( $+2 \mathrm{~mm}$ or less). Following the visual analog scale patients completed the comfort survey for only a single instance. When all procedures were completed for the first eye, the second eye was dosed with the other medication by the unmasked dosing coordinator, and all post-dosing procedures performed for the first eye were measured again for the second eye.

After all comfort evaluations were finished, Goldmann applanation tonometry was measured in both eyes. Also, a global product preference was collected. This test provided the patient a choice of which product (identified only as product \#1 or \#2) was most comfortable, or alternatively, that there was no preference between the two. In addition, any adverse events were recorded.

\section{Statistics}

The data was analyzed by PRN Pharmaceutical Research Network, LLC. All data analyses were two-sided and had an $\alpha$-level of $0.05 .{ }^{9,10}$ 
The primary safety variable was the VAS measured immediately after dosing. The visual analog scale used for this study (Product Comfort Preference Scale ${ }^{\odot}$, Alcon Laboratories) is scored from $0-100$ with ' 0 ' representing no pain and ' 100 ' being the greatest possible pain. The test consists of a simple horizontal which represents their perceived pain from ' 0 ' (perfect comfort) to ' 100 ' (the worst pain imaginable). The patient marks the point along the line that represents their perceived pain.

The study was sized based on a paired t-test model. Travoprost BAK-free was hypothesized to have a comfort level just above placebo and similar to brinzolamide (approximately $7 \mathrm{~mm}) .{ }^{10}$ In contrast, latanoprost was hypothesized to have an approximate comfort level twice as good as dorzolamide. ${ }^{11}$ These hypotheses supposed that approximately a $5 \mathrm{~mm}$ difference existed between groups. Consequently, if 51 patients completed the study, assuming a standard deviation of $10 \mathrm{~mm}$ for travoprost BAK-free and $22 \mathrm{~mm}$ for latanoprost, then the study had an $80 \%$ power to exclude a $5 \mathrm{~mm}$ difference between groups. ${ }^{12}$

The VAS immediately after dosing, the VAS at each timepoint following dosing apart from the initial measurement, and the comfort survey for each question (The Nine Point Comfort Survey $^{\odot}$, Alcon Laboratories) were analyzed by a one-way ANOVA test within a Matched Pairs platform to determine a significant difference between eyes. ${ }^{13}$ In addition, the peak measure, and the length of time until return to baseline on the VAS, also were analyzed by a one-way ANOVA test within a Matched Pairs platform. Apart from the primary endpoint, a Bonferroni correction was used to adjust the p-values for the three scales because of multiple measures. ${ }^{14}$ ANOVA evaluations were performed on all measured parameters both for the absolute levels as well as the changes from baseline. ${ }^{14}$

Slit lamp biomicroscopy and concomitant medications were described and not evaluated statistically. Visual acuity and intraocular pressure were analyzed between eyes by a one-way ANOVA test within a Matched Pairs platform. Adverse events were analyzed between eyes by a McNemar test. ${ }^{14}$ In contrast, patient global preference was evaluated by a chi-square test. ${ }^{14}$

\section{Results}

\section{Patients}

Patient characteristics for the 54 completed subjects are shown in Table 1. No unusual characteristics of our patient population for the United States were obvious. One additional subject was terminated from the study between Visits 1 and 2 because he was lost to follow-up.

\section{Comfort}

The primary endpoint showed that there was no significant difference at five seconds after dosing in mean comfort on the visual analogue scale between latanoprost $(7.1 \pm 16.2 \mathrm{~mm})$ and travoprost BAK-free $(7.8 \pm 16.1 \mathrm{~mm}, \mathrm{P}=0.53)$ above a common untreated baseline of $7.5 \pm 15.9 \mathrm{~mm}$.

Further, the mean peak level of discomfort was $8.4 \pm 16.5 \mathrm{~mm}$ for latanoprost and $10.5 \pm 17.0 \mathrm{~mm}$ for travoprost BAK-free $(\mathrm{P}=0.21)$. The most common time after dosing that the

Table I Patient characteristics

\begin{tabular}{|c|c|c|}
\hline Characteristic & $\mathbf{N}$ & Percentage \\
\hline \multicolumn{3}{|l|}{ A. Gender } \\
\hline Female & 37 & $68.5 \%$ \\
\hline Male & 17 & $31.5 \%$ \\
\hline \multicolumn{3}{|l|}{ B. Race } \\
\hline Caucasian & 42 & $77.8 \%$ \\
\hline Black & 9 & $16.7 \%$ \\
\hline Hispanic & 2 & $3.7 \%$ \\
\hline Middle Eastern & 1 & $1.9 \%$ \\
\hline \multicolumn{3}{|l|}{ C. Iris color } \\
\hline Hazel & 24 & $44.4 \%$ \\
\hline Brown & 20 & $37.0 \%$ \\
\hline Green & 6 & $11.1 \%$ \\
\hline Blue & 4 & $7.4 \%$ \\
\hline \multicolumn{3}{|l|}{ D. Medical history } \\
\hline Cardiovascular arterial hypertension & 39 & $72.2 \%$ \\
\hline Cardiovascular lipid disorders & 25 & $46.3 \%$ \\
\hline Endocrine diabetes & 19 & $35.2 \%$ \\
\hline \multicolumn{3}{|l|}{ E. Opthalmic history } \\
\hline Primary open-angle glaucoma & 35 & $64.8 \%$ \\
\hline Ocular hypertension & 16 & $29.6 \%$ \\
\hline Pigmentary glaucoma & 3 & $5.6 \%$ \\
\hline \multicolumn{3}{|l|}{ F. Glaucoma medication history } \\
\hline Latanoprost $0.005 \%$ & 29 & $35.2 \%$ \\
\hline Travoprost $0.004 \%$ & 18 & $33.3 \%$ \\
\hline Bimatoprost $0.03 \%$ & 6 & $11.1 \%$ \\
\hline Mean number of glaucoma medications & 1.82 & \\
\hline $\begin{array}{l}\text { Mean time on glaucoma medications } \\
\text { (in years) }\end{array}$ & 12.56 & \\
\hline \multicolumn{3}{|l|}{ G. Eyes assigned to each medicine } \\
\hline & OD & \\
\hline \multicolumn{3}{|l|}{ Test medication } \\
\hline Latanaprost & 25 & $46.3 \%$ \\
\hline \multirow[t]{2}{*}{ Travaprost BAK-free } & 29 & $53.7 \%$ \\
\hline & OS & \\
\hline Latanaprost & 29 & $53.7 \%$ \\
\hline Travaprost BAK-free & 25 & $46.3 \%$ \\
\hline
\end{tabular}


peak effect occurred was five seconds for latanoprost (range 5-600 seconds) and five seconds for travoprost BAK-free (range 5-120 seconds). The mean time required to return to baseline comfort was $16.8 \pm 84.1$ seconds for latanoprost and $6.7 \pm 23.7$ seconds for travoprost BAK-free $(\mathrm{P}=0.41)$. Further, at no time point following dosing was there a significant difference between treatments for the level of comfort ( $\mathrm{P}>0.05$; Please see Table 2). In addition, no differences were observed between products when classified as to severity by the baseline OSDI. The actual power to exclude a $5 \mathrm{~mm}$ difference between groups was $99.9 \%$ based on a standard deviation of $5 \mathrm{~mm}$, five seconds after dosing.

In addition, the comfort survey demonstrated no difference between products for burning, stinging, foreign body sensation, overall comfort and general acceptance of the product, both of absolute levels and changes from baseline (P > 0.05; see Table 3).

Following study medicine dosing and all comfort measures, the mean intraocular pressure for the travoprost BAK-free treated eye was $20.2 \pm 5.4 \mathrm{mmHg}$ and for the latanoprost-treated eye, $19.8 \pm 4.7 \mathrm{mmHg}(\mathrm{P}=0.22)$. Mean Snellen visual acuity was for the travoprost BAK-free-treated eye was $20 / 25.3 \pm 7.0$ and for the latanoprost-treated eye was $20 / 25.6 \pm 8.0(P=0.71)$. Latanoprost was preferred by 17 (31\%), travoprost BAK-free by 18 (33\%), and no preference was given by $19(35 \%)$ patients $(\mathrm{P}=0.92)$.

\section{Adverse effects}

Only one adverse event was noted in this single dose study. Hypertrichosis was noted in both eyes in one patient. This event was not believed by the investigator to be related to the study medicines. There were no serious adverse events during the study.

\section{Discussion}

A high percent of glaucoma patients suffer from chronic ocular surface diseases. ${ }^{11}$ Further, antiglaucoma medications are often associated with ocular adverse reactions such as dry eye, and burning or stinging sensations. ${ }^{15}$ These undesirable effects potentially might lead to treatment discontinuation and reduced quality of life. ${ }^{11}$

BAK is the preservative in the great majority of medicines to treat glaucoma. ${ }^{16}$ Recent research has noted, however, that BAK also may be toxic to the anterior segment epithelium

Table 2 Mean post-dosing readings on the product preference scale

\begin{tabular}{|c|c|c|c|c|}
\hline \multirow[b]{2}{*}{ Time (seconds) } & \multirow[b]{2}{*}{$\mathbf{N}$} & \multicolumn{3}{|l|}{ Mean } \\
\hline & & Latanoprost & Travoprost BAK-free & P-value \\
\hline 5 & 54 & $7.1 \pm 16.2$ & $7.8 \pm 16.1$ & 0.53 \\
\hline 10 & 54 & $6.8 \pm 15.4$ & $7.4 \pm 15.8$ & 0.55 \\
\hline 15 & 54 & $7.0 \pm 15.2$ & $7.2 \pm 15.3$ & 0.84 \\
\hline 20 & 54 & $6.8 \pm 15.2$ & $7.3 \pm 15.2$ & 0.58 \\
\hline 25 & 54 & $6.8 \pm 15.2$ & $7.0 \pm 15.2$ & 0.81 \\
\hline 30 & 54 & $7.0 \pm 15.5$ & $6.7 \pm 15.0$ & 0.73 \\
\hline 35 & 54 & $7.0 \pm 15.5$ & $6.4 \pm 15.0$ & 0.40 \\
\hline 40 & 54 & $6.8 \pm 15.2$ & $6.4 \pm 15.0$ & 0.47 \\
\hline 45 & 54 & $6.8 \pm 15.2$ & $6.4 \pm 15.0$ & 0.47 \\
\hline 50 & 54 & $6.8 \pm 15.2$ & $6.7 \pm 15.1$ & 0.90 \\
\hline 55 & 54 & $6.8 \pm 15.2$ & $6.6 \pm 15.1$ & 0.80 \\
\hline 60 & 54 & $6.8 \pm 15.2$ & $6.6 \pm 15.1$ & 0.71 \\
\hline 120 & 54 & $7.2 \pm 15.3$ & $7.6 \pm 16.4$ & 0.78 \\
\hline 300 & 54 & $6.9 \pm 15.2$ & $7.2 \pm 15.9$ & 0.80 \\
\hline 600 & 54 & $6.9 \pm 15.2$ & $6.5 \pm 15.4$ & 0.61 \\
\hline 900 & 54 & $6.8 \pm 15.2$ & $5.7 \pm 15.1$ & 0.06 \\
\hline 1200 & 54 & $6.8 \pm 15.2$ & $5.7 \pm 15.1$ & 0.06 \\
\hline 1800 & 54 & $6.7 \pm 15.2$ & $5.8 \pm I 5 . I$ & 0.11 \\
\hline Peak & 54 & $8.4 \pm 16.5$ & $10.5 \pm 17.0$ & 0.21 \\
\hline $\begin{array}{l}\text { Return to baseline } \\
\text { (seconds) }\end{array}$ & 54 & $16.8 \pm 84.1$ & $6.7 \pm 23.7$ & 0.41 \\
\hline
\end{tabular}


Table 3 Comfort survey results

\begin{tabular}{|c|c|c|c|c|c|}
\hline \multirow[b]{2}{*}{ Time (seconds) } & \multirow{2}{*}{$\frac{\text { Baseline }}{\text { Mean }}$} & \multicolumn{2}{|c|}{ Latanoprost } & \multicolumn{2}{|c|}{ Travoprost BAK-free } \\
\hline & & Mean & P-value & Mean & P-value \\
\hline NPCS burning & $7.5 \pm 15.9$ & $8.4 \pm 16.5$ & 0.37 & $10.5 \pm 17.0$ & 0.06 \\
\hline NPCS stinging & $0.2 \pm 0.8$ & $0.1 \pm 0.7$ & 0.18 & $0.1 \pm 0.7$ & 0.18 \\
\hline NPCS foreign body & $0.04 \pm 0.3$ & $0.02 \pm 0.1$ & 0.32 & $0.02 \pm 0.1$ & 0.32 \\
\hline NPCS general discomfort & $0.1 \pm 1.0$ & $0.02 \pm 0.1$ & 0.40 & $0.02 \pm 0.1$ & 0.40 \\
\hline NPCS overall accept & $0.5 \pm 1.1$ & $0.2 \pm 0.7$ & 0.04 & $0.3 \pm 1.1$ & 0.30 \\
\hline
\end{tabular}

Abbreviation: NPCS, Nine Point Comfort Survey.

of the eye. ${ }^{16}$ Consequently, the development of antiglaucoma medicines that are preserved without BAK might help reduce anterior segment symptoms in patients.

Travoprost BAK-free was recently released commercially as the first non-BAK-preserved prostaglandin. Baudouin has noted in an in-vitro conjunctival cell line that the travoprost BAK-free solution did not demonstrate the toxic effects of BAK-preserved prostaglandin preparations or BAK alone. ${ }^{17}$ Further, Yee and colleagues have shown reduced in-vitro corneal cell toxicity with travoprost BAK-free as opposed to the commercial latanoprost preparation. ${ }^{18}$ In addition, Kahook and Noecker demonstrated in rabbits less deleterious effects on the ocular surface with non-BAK-preserved medications. ${ }^{19}$ In patients, the presence of BAK in prostaglandin formulations has been associated with more anterior segment side effects than those treated with the travoprost BAK-free preparation. ${ }^{20}$

The primary objective of this study was to determine the short-term comfort after a single dose of travoprost BAK-free compared to latanoprost in patients with primary open-angle glaucoma or ocular hypertension.

This study showed that the endpoint, the VAS at five seconds after dosing, showed only very mild discomfort and no significant difference between latanoprost $(7.1 \pm 16.2 \mathrm{~mm})$ and travoprost BAK-free $(7.8 \pm 16.1 \mathrm{~mm})$.

Further, secondary safety variables showed no differences between treatments following dosing for discomfort at any individual time point, time of peak discomfort, or the time required to return to baseline comfort. In addition, the comfort survey demonstrated no difference between products for burning, stinging, foreign body sensation, overall comfort and general acceptance of the product, by both absolute levels and changes from baseline.

Since BAK is known to have toxic effects on the anterior surface of the eye, a difference in comfort might have been anticipated between a glaucoma medicine containing a high percentage of BAK (latanoprost) and one without (travoprost BAK-free). The reason for a lack of difference in comfort between the study medicines is not known precisely by our results but may have been derived from the following. First, latanoprost is known as a fairly comfortable medicine despite its higher concentration of BAK. Accordingly, Stewart and colleagues noted in healthy individuals only minimal differences in anterior segment staining with latanoprost following three days of dosing to untreated baseline. ${ }^{21}$ A longer-term dosing trial with latanoprost may be needed to demonstrate a difference in comfort favoring travoprost BAK-free.

Second, as this current study was a single-dose comparison in patients who had been washed out of their active medication for at least three days, the surface may have recovered from any anterior segment toxic effects associated with the prior glaucoma medicine. Consequently, a single dose of medication, even with BAK present, may not have had a sufficient impact to differentiate comfort between products on the relatively healthy epithelium. In a longer term study chronically dosed BAK may demonstrate a greater likelihood to induce inflammation and subsequent apoptosis, causing clinical symptoms and an irritated anterior segment. In such a clinical setting, differentiation of comfort between the two study medications, one with and one without BAK, may have a greater likelihood of success consistent with several past studies. ${ }^{20}$

This study suggests that following a single drop, both latanoprost and travoprost BAK-free exhibit similar levels of very transient and mild discomfort.

This study did not evaluate chronic dosing of latanoprost versus travoprost BAK-free on comfort. As mentioned above, longer-term dosing may have found clinical differences in signs or symptoms between a BAK preserved solution and one without. In addition, this study did not evaluate quality of life and compliance in patients treated with a BAK-preserved and -free solutions. Any glaucoma medication that produces chronic irritation ${ }^{11}$ potentially may more likely cause noncompliance $^{23}$ and problems with activities of daily living. ${ }^{24}$ 
More research is needed generally to fully define the impact of BAK on patients' lives and to further develop medications which can be dosed chronically without a harsh active preservative and maintain sterility.

\section{Disclosure}

This study was sponsored by an unrestricted grant from Alcon Laboratories, Inc.

\section{References}

1. Stewart WC. Clinical Practice of Glaucoma. Thorofare, NJ: SLACK, Inc; 1990.

2. Baudouin C. Allergic reaction to topical eyedrops. Curr Opin Allergy Clin Immunol. 2005;5:459-463.

3. Ishibashi $\mathrm{T}$, Yokoi N, Kinoshita S. Comparison of the short-term effects on the human corneal surface of topical timolol maleate with and without benzalkonium chloride. J Glaucoma. 2003;12:486-490.

4. Katz LJ. Twelve-month evaluation of brimonidine-purite versus brimonidine in patients with glaucoma or ocular hypertension. J Glaucoma. 2002;11:119-126.

5. Lewis RA, Katz GJ, Weiss MJ, et al; Travoprost BAC-free Study Group. Travoprost $0.004 \%$ with and without benzalkonium chloride: a comparison of safety and efficacy. J Glaucoma. 2007;16:98-103.

6. Stewart WC, Pfieffer N, Mathis HM. Meta-analysis of articles evaluating routine intraocular pressure control for monotherapy in the United States and Germany. Eur J Ophthalmol. 2009. In press.

7. Camras CB, Alm A, Watson P, Stjernschantz J. Latanoprost, a prostaglandin analog, for glaucoma therapy. Efficacy and safety after 1 year of treatment in 198 patients. Latanoprost Study Groups. Ophthalmology. 1996;103:1916-1924.

8. Schiffman RM, Christianson MD, Jacobsen G, Hirsch JD, Reis BL. Reliability and validity of the Ocular Surface Disease Index. Arch Ophthalmol. 2000;118:615-621.

9. Rosner B. Fundamentals of Biostatistics. Boston, MA: Duxbury Press; 1982. p. 301-304.

10. Stewart WC, Day DG, Stewart JA, Holmes KT, Jenkins JN. Short-term ocular tolerability of dorzolamide $2 \%$ and brinzolamide $1 \%$ versus placebo in primary open-angle glaucoma and ocular hypertension subjects. Eye. 2004;18:905-910.
11. Alm A, Camras CB, Watson PG. Phase III latanoprost studies in Scandinavia, the United Kingdom and the United States. Surv Ophthalmol. 1997;41(Suppl 2):S105-S110.

12. Siegel S. Non Parametric Statistics. Series in Psychology. New York, NY: McGraw Hill; 1956. p. 63-67.

13. Stewart WC, Stewart JA, Leech JN. Acute and chronic ocular symptoms of dorzolamide 2\% compared with placebo. J Glaucoma. 2003; 12:151-155.

14. Book SA. Essentials of Statistics. New York, NY: McGraw Hill Book Company; 1978.

15. Leung EW, Medeiros FA, Weinreb RN. Prevalence of ocular surface disease in glaucoma patients. J Glaucoma. 2008;17:350-355.

16. Baudouin C. Detrimental effect of preservatives in eyedrops: implications for the treatment of glaucoma. Acta Ophthalmol. 2008;86:716-726.

17. Baudouin C, Riancho L, Warnet J-M, Brignole F. In vitro studies of antiglaucomatous prostaglandin analogues: Travoprost with and without benzalkonium chloride and preserved latanoprost. Invest Ophthalmol Vis Sci. 2007;48:4123-4128.

18. Yee RW, Norcom EG, Zhao XC. Comparison of the relative toxicity of travoprost $0.004 \%$ without benzalkonium chloride and latanoprost $0.005 \%$ in an immortalized human cornea epithelial cell culture system. Adv Ther. 2006;23:511-519.

19. Kahook M, Noecker RJ. Comparison of corneal and conjunctival changes after dosing of travoprost preserved with sofZia, latanoprost with $0.02 \%$ benzalkonium chloride, and preservative-free artificial tears. Cornea. 2008;27:339-343.

20. Henry JC, Peace JH, Stewart JA, Stewart WC. Efficacy, safety, and improved tolerability of travoprost BAK-free ophthalmic solution compared with prior prostaglandin therapy. Clin Ophthalmol. 2008;2:613-621.

21. Stewart WC, Stewart JA, Leech JN, Jackson AL. Corneal punctate staining with latanoprost, bimatoprost and travoprost. J Glaucoma. 2003; $12: 475-479$

22. McCarey B, Edelhauser H. In vivo corneal epithelial permeability following treatment with prostaglandin analogs with or without benzalkonium chloride. J Ocul Pharmacol Ther. 2007;23:445-451.

23. Day DG, Sharpe ED, Atkinson MJ, Stewart JA, Stewart WC. The clinical validity of the treatment satisfaction survey for intraocular pressure in ocular hypertensive and glaucoma patients. Eye. 2006;20:583-590.

24. Freeman EE, Muñoz B, West SK, Jampel HD, Friedman DS. Glaucoma and quality of life: the Salisbury Eye Evaluation. Ophthalmology. 2008;115:233-238 